\title{
Aggressive haematological malignancies
}

\author{
Andrew Wotherspoon ${ }^{1}$
}

Received: 7 August 2017 / Accepted: 8 August 2017 /Published online: 16 August 2017

(C) Springer-Verlag GmbH Deutschland 2017

In this issue of the Journal, there is a miniseries that covers difficult and controversial topics in the diagnosis of aggressive haematological malignancies. The three articles summarise current knowledge and reflect changes in the classification of these tumours in the context of the updated 2016 World Health Organisation (WHO) classification of haematolymphoid malignancies. The authors have used cases submitted to the workshop of the 18th meeting of the European Association for Haematopathology held in Basel, Switzerland, in 2016 as the basis for their discussion of the pertinent issues for each group of lymphomas.

The reviews in this miniseries include the following:

- Histiocytic and dendritic cell neoplasms: what have we learnt by studying 67 cases

- B cell lymphomas with discordance between pathological features and clinical behaviour

- The clinicopathologic spectrum of mature aggressive B cell lymphomas

In the article covering histiocytic and dendritic cell tumours, Facchetti et al. highlight current concepts in this group of rare tumours. The terminology and classification used in the article are based on the WHO classification rather than the other scheme that is in current usage developed by the Histiocyte Society and includes histiocytic sarcoma, Langerhans cell

This article is part of the Topical Collection on Aggressive B-cell lymphomas and histiocytic neoplasias

Andrew Wotherspoon

Andrew.Wotherspoon@rmh.nhs.uk

1 Department of Histopathology, Royal Marsden Hospital, 203 Fulham Road, Chelsea, London SW3 6JJ, UK histiocytosis, Langerhans cell sarcoma, indeterminate dendritic cell sarcoma, interdigitating cell sarcoma, follicular dendritic cell sarcoma, fibroblastic reticular cell tumour, disseminated juvenile xanthogranuloma, Erdheim-Chester disease and blastic plasmacytoid dendritic cell neoplasm.

The authors present a summary of available data on the molecular studies and prognostic indicators in these rare tumours. Included in the article is confirmation of the overexpression of PD-L1 expression in these tumours as well as the first report of expression of CD30 which may indicate a potential role for checkpoint inhibitors and anti-CD30 targeted therapy in the management of these neoplasms.

The second article from de Leval et al. covers the topic of B cell lymphomas in which the morphological appearances do not predict the clinical behaviour. This includes lymphomas with a morphological appearance that would normally be associated with indolent behaviour, but the outcome is worse than predicted as well as rare cases of lymphomas with features that would normally indicate an aggressive clinical course but where the true outcome is favourable. The subject of transformation is covered in the context of indolent lymphomas as well as the topic of large B cell lymphomas that arise in specific locations and are associated with indolent behaviour, particularly those associated with cardiac thrombosis/prostheses and lymphomas arising in states of fluid overload.

In the third article, Rimza et al. covers the topic of mature aggressive $\mathrm{B}$ cell lymphomas. This is an area that has shown significant changes in the updated 2016 WHO classification compared to previous editions. This reflects increased understanding over the roles of translocations involving $M Y C$ and $B C L 2$ and/or BCL6 genes (double- and triple-hit lymphomas). The new terminology is used alongside previous groupings to highlight the correct criteria for each group on the basis of morphology and genetic characteristics. In particular, the challenging question of which strategy to adopt for identifying 
cases to be assessed for these translocations is addressed. The authors also summarise cases of Burkitt lymphoma with unusual clinical features including spontaneous remission and underlying immunosuppression as well as the recently recognised Burkitt-like lymphoma with 11q aberration.
Compliance with ethical standards Not applicable.

Funding None to declare.

Conflict of interest Not applicable. 